\title{
SENSILLES ET PROTONÉPHRIDIES DES CERCAIRES DE CENTROCESTUS FORMOSANUS ET CENTROCESTUS SP (Centrocestinae, Heterophyidae)
}

\author{
Ch. BAYSSADE-DUfOUR*, J.-L. ALBARET* et C. K. OW-YANG**
}

\begin{abstract}
RÉSUMÉ. Une description est donnée de la chétotaxie de deux cercaires de Centrocestinae (Heterophyidae, Opisthorchioidea) de Malaisie : Centrocestus formosanus et Centrocestus sp. - La comparaison avec la chétotaxie de cercaires systématiquement apparentées semble indiquer que $1^{\circ} \mathrm{La}$ superfamille des Opisthorchioidea constitue un groupe naturel dont les Centrocestinae font partie

$2^{\circ}$ Les Centrocestinae diffèrent cependant des autres sous-familles d'Heterophyidae. Cette famille se révèle hétérogène, elle ne peut pas être clairement définie d'un point de vue chétotaxique.
\end{abstract}

\section{Sensillae and flame cells of cercariae of Centrocestus formosanus and Centrocestus sp. (Centrocestinae, Heterophyidae).}

SUMMARY. A description is given of cercarial chaetotaxy of two Centrocestinae (Heterophyidae, Opisthorchioidea) from Malaysia: Centrocestus formosanus and Centrocestus $\mathrm{sp}$.-Comparison with cercarial chaetotaxy of taxonomically related groups indicates that

10 The chaetotaxy of the Centrocestinae agrees with that of other Opisthorchioidea,

$2^{\circ}$ There is an important difference between chaetotaxy of Centrocestinae and other Heterophyidae which suggest that the family is heterogeneous.

La famille des Heterophyidae Odhner peut se caractériser par la présence chez l'adulte de trois ventouses, la troisième ventouse étant le gonotyl ou ventouse génitale. L'une des sous-familles qu'elle comprend : les Centrocestinae, regroupe des genres possédant un gonotyl de taille modeste et des épines péribuccales, mais leur cycle biologique est semblable à celui des autres Heterophyidae : des Mollusques Prosobranches sont les premiers hôtes, plusieurs genres de Poissons, rarement des Batraciens, sont les seconds hôtes, des Oiseaux et des Mammifères piscivores sont les hôtes définitifs.

Le genre Centrocestus comprend des espèces déterminées d'après leur morphologie (sinon de tous les stades de l'œuf à l'adulte, du moins du stade adulte mûr) et

* Muséum National d'Histoire Naturelle, Laboratoire de Zoologie-Vers, associé au CNRS, 61 rue Buffno, F 75231 Paris Cedex 05.

** Institute for Medical Research, Jalan Pahang, Kuala Lumpur 02-14 Malaysia.

Accepté le 28 avril I982. 
d'après leur localisation (sinon dans les trois hôtes successifs, du moins dans l'hôte définitif). Il comprend aussi des espèces indéterminées décrites d'après des métacercaires pourvues d'une spinulation et d'un appareil génital non ovigère, aucun adulte n'ayant pu être obtenu à partir de ces métacercaires (Ito, 1964).

Le premier lot de cercaires considérées dans ce travail provient d'un Melanoides tuberculata naturellement infesté de Kampong Pangland, Negeri Sembilan (Malaisie) ; ces cercaires se sont enkystées sur des Poissons et les métacercaires obtenues se sont transformées en adultes après ingestion par un Rat de Laboratoire. L'espèce est identique à/ou jumelle de Centrocestus formosanus, dont les cercaires sont parasites de Semisulcospira libertina à Formose et les adultes parasites de l'Homme (Nishigori, 1924).

Le second lot de cercaires considérées est indéterminé ; elles sont émises par un Melanoides tuberculata naturellement infesté, également originaire de Kampong Pangland, Negeri Sembilan (Malaisie) ; elles se sont enkystées chez un Poisson et se sont transformées en métacercaires identifiables à Centrocestus; nous les appelons Centrocestus sp.

Les cellules-flammes de ces deux lots de cercaires : $C$. formosanus et $C$. sp., imprégnées au protéinate d'argent selon la méthode d'Albaret (1973), sont au nombre de 8 par hémicorps, soit $(2+2)+(2+2)$, ce qui est conforme à ce que l'on connaît chez $C$. formosanus et chez toutes les cercaires de Centrocestus actuellement décrites (Martin, 1958 ; Yamaguti, 1938 et 1975) (fig. 1, A, B, C).

Les autres éléments de la morphologie de ces deux cercaires : glandes de pénétration, appareil digestif, vessie, etc., sont identiques, et rien, sauf les sensilles, ne peut les différencier à ce stade. Les sensilles imprégnées au nitrate d'argent selon la méthode de Combes et coll. (1976) sont désignées selon la nomenclature créée par Richard (1971).

\section{Chétotaxie de la cercaire de Centrocestus formosanus}

- Région céphalique (fig. 1,D E) :

Elle porte 66 à 72 sensilles réparties selon cinq cycles, soit de part et d'autre du plan médio-sagittal :

$$
\begin{aligned}
& \mathrm{C}_{\mathrm{I}}=1 \mathrm{C}_{\mathrm{I}} 1,11 \text { à } 12 \mathrm{C}_{\mathrm{I}} 2 \\
& \mathrm{C}_{\mathrm{II}}=1 \mathrm{C}_{\mathrm{II}} 1,3 \mathrm{C}_{\mathrm{II}} 2,2 \mathrm{C}_{\mathrm{II}} 3,2 \text { ou } 1 \mathrm{C}_{\mathrm{II}} 4 \\
& \mathrm{C}_{\mathrm{III}}=2 \mathrm{C}_{\mathrm{II}} 1,2 \mathrm{C}_{\mathrm{III}} 2,3 \mathrm{C}_{\mathrm{II}} 3 \\
& \mathrm{C}_{\mathrm{IV}}=2 \mathrm{C}_{\mathrm{IV}} 1,1 \mathrm{C}_{\mathrm{IV}} 2 \\
& \mathrm{C}_{\mathrm{V}}=2 \mathrm{C}_{\mathrm{V}} 1,0 \text { ou } 1 \mathrm{C}_{\mathrm{V}} 2,2 \mathrm{C}_{\mathrm{V}} 3
\end{aligned}
$$

- Région corporelle (fig. $2 A, B, C, D$ ) :

Elle porte 32 à 42 sensilles, soit par hémicorps :

$$
\begin{aligned}
& \mathrm{A}_{\mathrm{I}}=2 \mathrm{~A}_{\mathrm{I}} \mathrm{V}, 3 \text { à } 4 \mathrm{~A}_{\mathrm{I}} \mathrm{L}, 1 \mathrm{~A}_{\mathrm{I}} \mathrm{D} \\
& \mathrm{A}_{\mathrm{II}}=1 \mathrm{~A}_{\mathrm{II}} \mathrm{V}, 1 \text { à } 2 \mathrm{~A}_{\mathrm{II}} \mathrm{L}, 2 \text { ou } 3 \mathrm{~A}_{\mathrm{II}} \mathrm{D}
\end{aligned}
$$



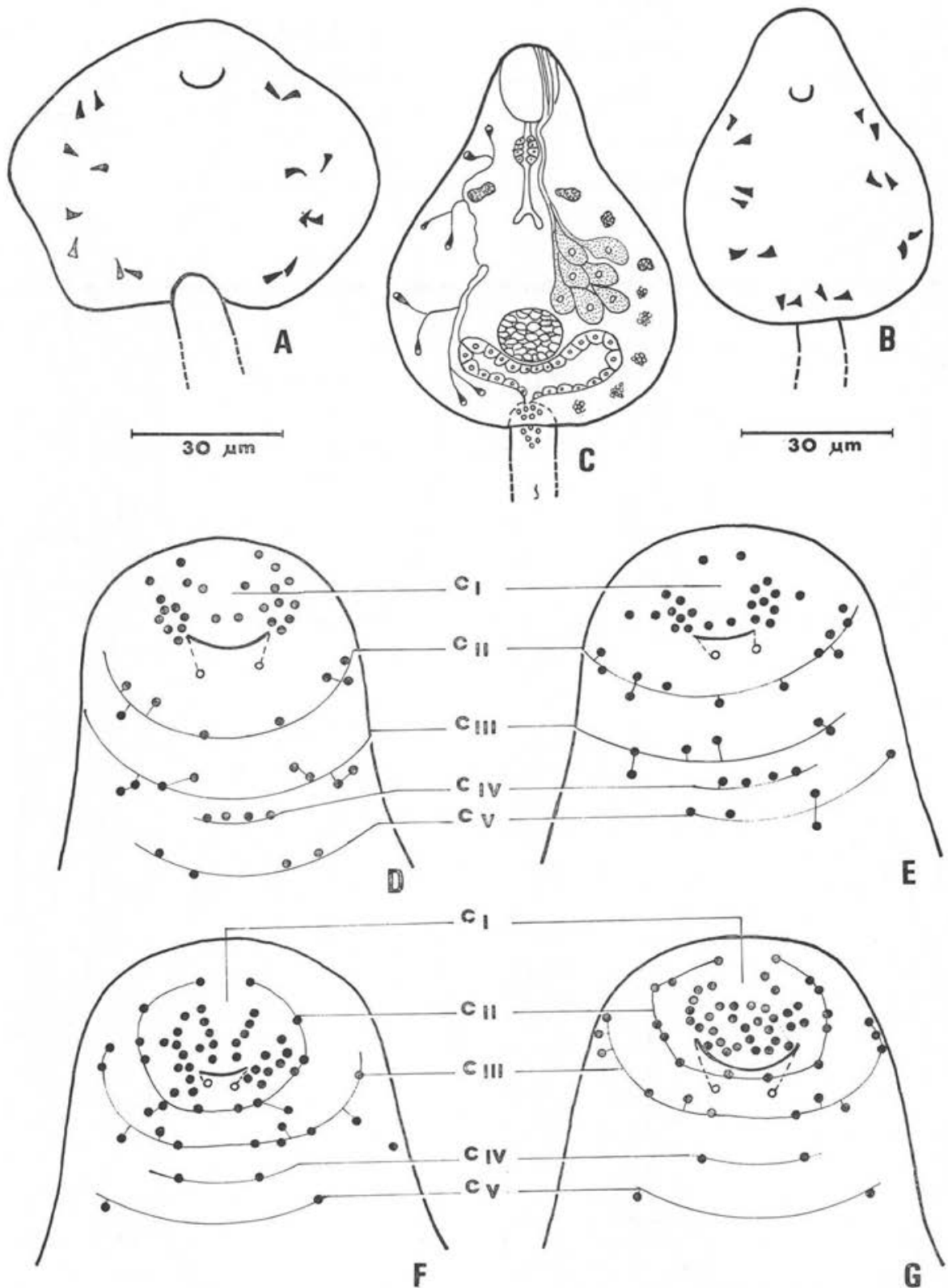

Fig. I. - Cercaires de Centrocestus formosanus et Centrocestus sp.

A) Centrocestus formosanus : cellules flammes imprégnées au protéinate d'argent ; B) Centrocestus sp. : cellules flammes imprégnées au protéinate d'argent; C) Morphologie générale de C. formosanus d'après Martin (I958) ; D-E) Centrocestus formosanus : chétotaxies céphaliques ventrales, détail ; F-G) Centrocestus sp. : chétotaxies céphaliques ventrales, détail. 

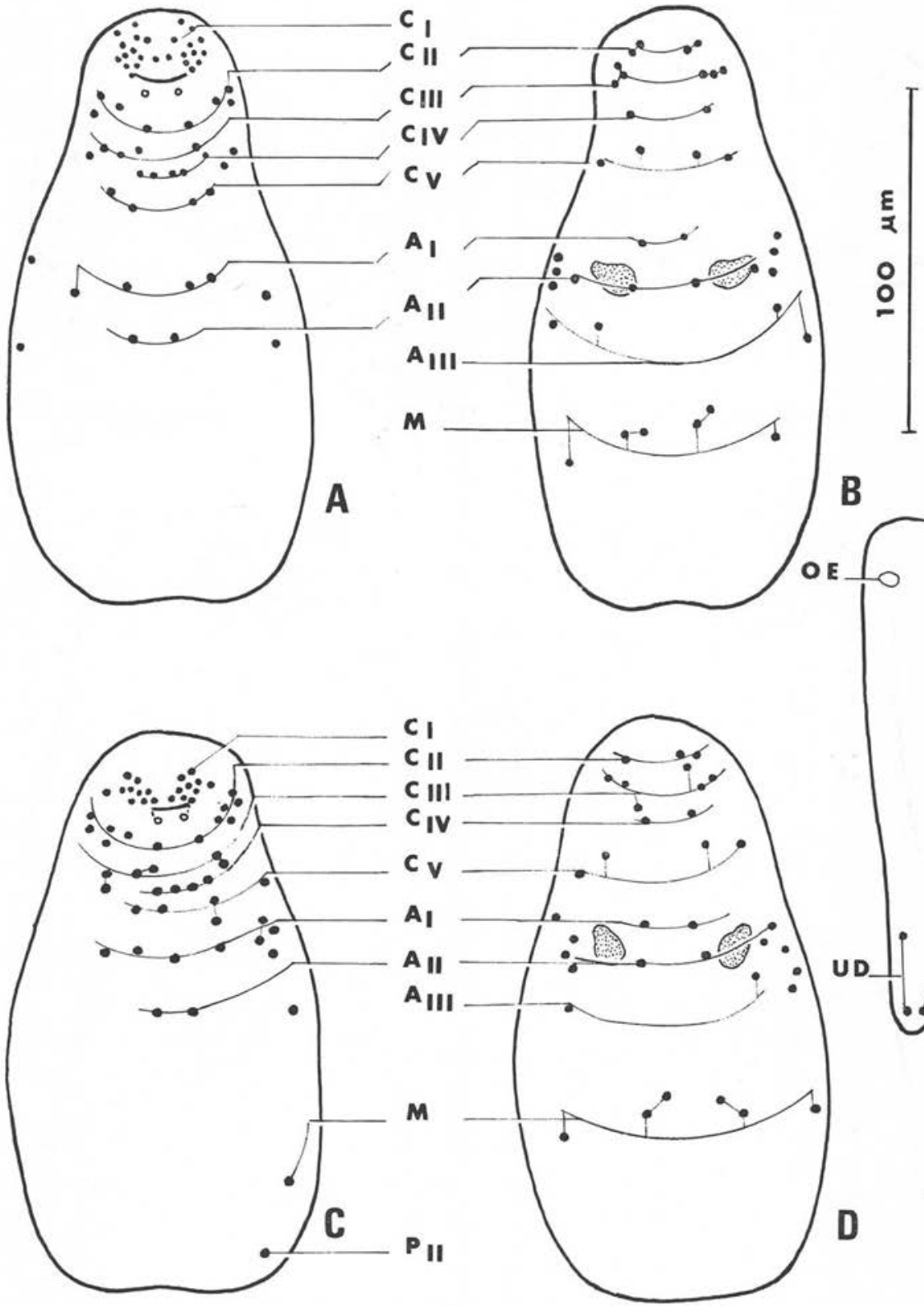

Fig. 2. - Cercaires de Centrocestus formosanus.

A-C) Chétotaxies céphaliques et corporelles ventrales. B-D) Chétotaxies céphaliques et corporelles dorsales. E) Chétotaxie caudale. 


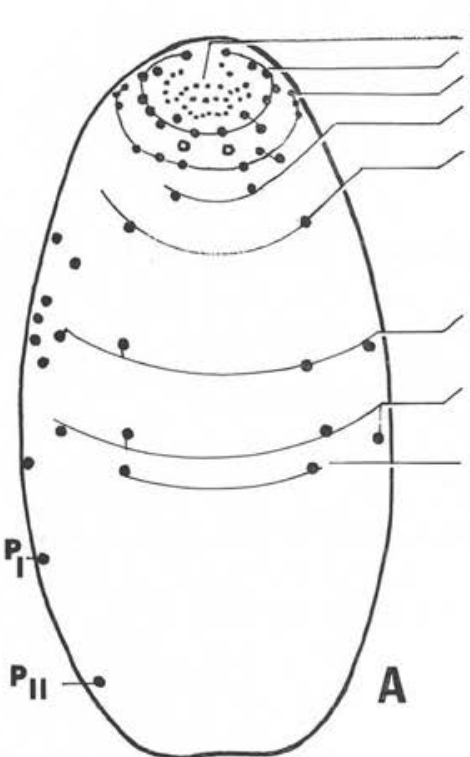

c.

cliI

A

A II

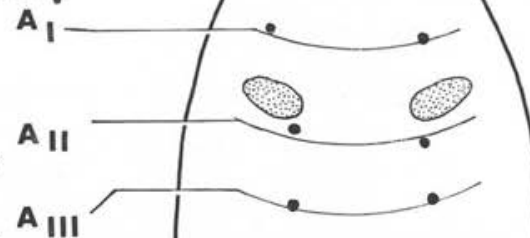

$\frac{\xi}{5}$

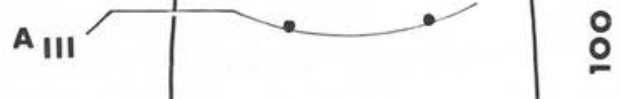

A IV

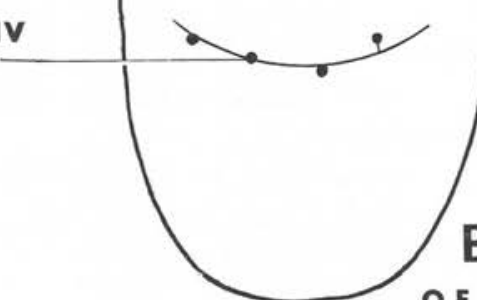

B
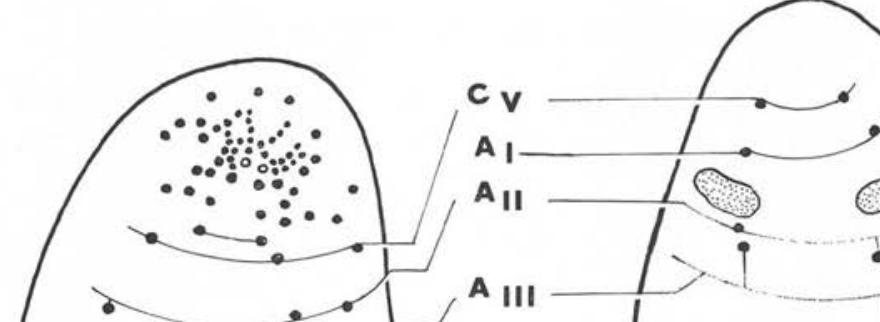

A III
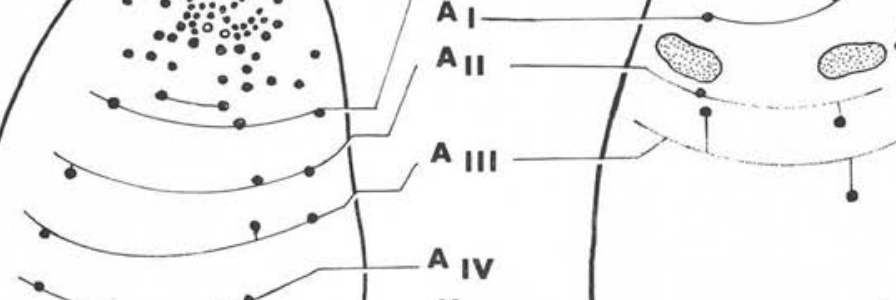

A IV

•

M

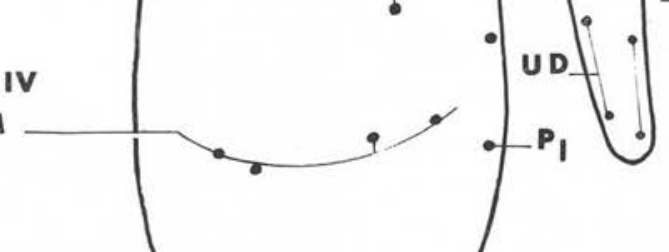

Fig. 3. - Cercaires de Centrocestus sp.

A-C) Chétotaxies céphaliques et corporelles ventrales. B-D) Chétotaxies céphaliques et corporelles dorsales. E) Chétotaxie caudale. 


$$
\begin{aligned}
& \mathrm{A}_{\text {III }}=0 \text { ou rarement } 1 \mathrm{~A}_{\mathrm{III}} \mathrm{V}, 1 \text { ou } 2 \mathrm{~A}_{\mathrm{III}} \mathrm{D} \\
& \mathrm{M}=1 \mathrm{ML}, 3 \mathrm{MD} \\
& \mathrm{P}_{\mathrm{II}}=1 \mathrm{P} \mathrm{P}
\end{aligned}
$$

- Quene (fig. 2, E):

$\mathrm{U}=2$ paires de UD

Le nitrate imprègne aussi, dans la région proximale de la queue, les orifices excréteurs.

\section{Chétotaxie de la cercaire de Centrocestus sp.}

- Région céphalique (fig. $1 \mathrm{~F}, \mathrm{G}$ ) :

Elle porte 62 à 70 sensilles réparties selon cinq cycles, soit de part et d'autre du plan médio-sagittal :

$$
\begin{aligned}
& \mathrm{C}_{\mathrm{I}}=1 \mathrm{C}_{\mathrm{I}} 1,15 \mathrm{C}_{\mathrm{I}} 2, \text { exceptionnellement } 13,14 \text { ou } 16 \\
& \mathrm{C}_{\mathrm{II}}=1 \mathrm{C}_{\mathrm{II}} 1,2 \mathrm{C}_{\mathrm{II}} 2,2 \mathrm{C}_{\mathrm{II}} 3,2 \mathrm{C}_{\mathrm{II}} 4 \\
& \mathrm{C}_{\mathrm{III}}=3 \mathrm{C}_{\mathrm{III}} 1,3 \mathrm{C}_{\mathrm{III}} 2 \\
& \mathrm{C}_{\mathrm{IV}}=1 \mathrm{C}_{\mathrm{IV}} 1,1 \mathrm{C}_{\mathrm{IV}} 2 \\
& \mathrm{C}_{\mathrm{V}}=1 \mathrm{C}_{\mathrm{V}} 1,0 \text { ou } 1 \mathrm{C}_{\mathrm{V}} 2,1 \mathrm{C}_{\mathrm{V}} 3
\end{aligned}
$$

- Régions corporelle (fig. $3 \mathrm{~A}, \mathrm{~B}, \mathrm{C}, \mathrm{D}$ ) :

Elle porte 32 à 36 sensilles, soit de part et d'autre du plan médio-sagittal :

$$
\begin{aligned}
& \mathrm{A}_{\mathrm{I}}=1 \mathrm{~A}_{\mathrm{I}} \mathrm{L}, 1 \mathrm{~A}_{\mathrm{I}} \mathrm{D} \\
& \mathrm{A}_{I I}=1 \text { ou } 2 \mathrm{~A}_{I I} \mathrm{~V}, 4 \mathrm{~A}_{\mathrm{II}} \mathrm{L}, 1 \mathrm{~A}_{\mathrm{II}} \mathrm{D} \\
& \mathrm{A}_{\mathrm{III}}=1 \text { ou } 2 \mathrm{~A}_{\mathrm{III}} \mathrm{V}, 1 \mathrm{~A}_{\text {III }} \mathrm{D} \\
& \mathrm{A}_{\mathrm{IV}}=1 \mathrm{~A}_{\mathrm{IV}} \mathrm{V}, 1 \mathrm{~A}_{\mathrm{IV}} \mathrm{L} \\
& \mathrm{M}=2 \mathrm{MD} \\
& \mathrm{P}_{\mathrm{I}}=1 \mathrm{P}_{\mathrm{I}} \mathrm{L} \\
& \mathrm{P}_{\mathrm{II}}=1 \mathrm{P}_{\mathrm{II}} \mathrm{L} \\
& \text { - Queue (fig. 3, E): } \\
& \mathrm{U}=2 \text { paires de UD }
\end{aligned}
$$

Le nitrate imprègne aussi les orifices excréteurs caudaux.

\section{Discussion}

Les deux cercaires étudiées ont en commun les sensilles $\mathrm{C}_{\mathrm{I}} 1, \mathrm{C}_{\mathrm{II}} 1, \mathrm{C}_{\mathrm{II}} 3, \mathrm{C}_{\mathrm{II}} 4$, $\mathrm{C}_{\mathrm{IV}} 2, \mathrm{C}_{\mathrm{V}} 2, \mathrm{~A}_{\mathrm{II}} \mathrm{D}, \mathrm{P}_{\mathrm{II}} \mathrm{L}$ et UD ; elles diffèrent par le nombre des sensilles $\mathrm{C}_{\mathrm{I}} 2, \mathrm{C}_{\mathrm{II}} 2, \mathrm{C}_{\mathrm{III}} 1$ et $2, \mathrm{C}_{\mathrm{rv}} 1, \mathrm{C}_{\mathrm{V}} 1$. La chétotaxie cercarienne confirme donc les observations concernant la morphologie de la métacercaire et de l'adulte : nous avons affaire à deux espèces distinctes du genre Centrocestus, ou du moins à deux espèces distinctes de la sousfamille des Centrocestinae. 
Nous connaissons la chétotaxie de quelques cercaires appartenant à trois des dix sous-familles constituant la famille des Heterophyidae (Baer et Joyeux, in Grassé 1961) ; les espèces en cause sont :

- chez les Centrocestinae : $C$. formosanus et $C$. sp. (cf. ci-dessus) ;

- chez les Apophallinae : Apophallus donicus et A. muehlingi (Odening, 1973), Euryhelmis squamula (Combes, Jourdane et Richard, 1974) ;

- chez les Haplorchiinae : Haplorchis pumilio (Bayssade-Dufour et Ow-Yang, 1975).

Nous avons cherché des éléments communs à ces espèces, représentant trois sousfamilles, pour tenter de définir par voie chétotaxique la famille des Heterophyidae. Le résultat est négatif : les Centrocestinae s'opposent clairement aux autres Heterophiydae; ils ont, en particulier, 2 paires de papilles caudales, alors que les cercaires des deux autres sous-familles en ont 4 ou 5 paires. A notre point de vue, la famille des Heterophyidae telle qu'elle est actuellement constituée n'est donc pas homogène.

Outre les Heterophyidae, la superfamille des Opisthorchioidea comprend les Acanthostomidae et les Opisthorchiidae, dont quelques chétotaxies cercariennes sont connues, et d'autres familles dont la chétotaxie n'est pas connue. Les chétotaxies connues d'Ancathostomidae sont celles de :

- Acanthostomum imbutiforme (Maillard, 1973) ;

- Timoniella praeteritum (Bayssade-Dufour, 1979).

La chétotaxie connue d'Opisthorchiidae est celle de :

- Clonorchis sinensis (Bayssade-Dufour, Albaret, Dong Cham Kim, 1982).

Toutes ces cercaires d'Opisthorchioidea ont en commun une chétotaxie caudale de 4 à 5 paires de papilles, à l'exception des Centrocestinae, qui n'ont que 2 paires. Pourtant, l'ensemble des Opisthrochioidea, Centrocestinae compris, se caractérise aisément d'un point de vue chétotaxique : c'est le seul groupe de Digènes actuellement connu qui ait perdu sa symétrie hexaradiée sur le cycle $\mathrm{C}_{\mathrm{I}}$.

En conclusion, en ne considérant que les seuls éléments chétotaxiques, il apparaît que :

1 - La famille des Heterophyidae ne peut être clairement définie parmi les autres Opisthorchioidea, étant donné le caractère particulier des Centrocestinae.

2 - La superfamille des Opisthorchioidea constitue cependant un groupe naturel dont font partie les Centrocestinae.

\section{BIBLIOGRAPHIE}

Albaret J. L. : Imprégnation des formations ciliaires de l'appareil excréteur des cercaires par le protéinate d'argent. C.R. Acad. Sci. Paris, sér. D, I973, 276, 1453-1454.

Baer J., Joyeux Ch. : Classe des Trématodes. In : Grassé P. P., Traité de Zoologie, Masson, Paris, 196r, $4(\mathrm{I}), 56 \mathrm{r}-677$.

BAysSADE-DUfour Ch. : L'appareil sensoriel des cercaires et la systématique des Trématodes digénétiques. Mém. Mus. Natn. Hist. nat., série, A. I979, Zool., 113, I-81.

Bayssade-Dufour Ch, Albaret J. L., Dong Cham Kim : Chétotaxie cercarienne d'une souche coréenne de Clonorchis sinensis. Présence vraisemblable de ce Trém tode en Afrique. Ann. Parasitol. Hum. Comp., I982 (à paraître). 
BAyssade-Dufour Ch,. Ow-YANG C. K. : The sensory receptors of two cercariae from Malaysia : Trichobilharzia brevis (Schistosomatidae) and Haplorchis pumilio (Heterophyidae). The Southeast Asian J. Trop. Med. Pub. health, 1975, 6, 338-342.

Combes C., Bayssade-Dufour Ch., CAssone J. : Sur l'imprégnation et le montage des cercaires pour l'étude chétotaxique. Ann. Parasitol. Hum. Comp., I976, s1, 399-400.

COMBES C., JOURDANE J., RICHARD J. : Recherches sur le cycle biologique de Euryhelmis squamula (Rudolphi, I8I9) parasite de Neomys fodiens dans les Pyrénées. Z. Parasitkde, 1974, 44, 81-92.

Iro J. : Metagonimus and other human Heterophyid Trematodes. Progress of Medical Parasitology in Japan, 1964, $1,317-393$.

MAILlARD C.: Etude du cycle évolutif du Trématode : Acanthostomum imbutiforme (Molin, I859) Gohar, 1934, parasite de Morone labrax (Linné, 1758). Ann. Parasitol. Hum. Comp., 1973, $48,33-46$.

Martin W. E. : The life histories of some Hawaian Heterophyids Trematodes. J. Parasitol., 1958, $44,305-318$.

NishigorI M. : On a new trematode Stamnosoma formosanum n. sp. and its life history. Taiwan Igakkai Zasshi, 1924, 234, 181-228.

Odening K. : Der Lebenszyklus des Trematoden Apophallus donicus in Berlin im Wergleich zu A. muchlingi. Biol. Zbl., 1973, 92, 455-494.

Richard J. : La chétotaxie des cercaires. Valeur systématique et phylétique. Mém. Mus. Natn. Hist. nat., sér. A, I971, Zool. 67, I-I79.

YAMAGUTI S. : Zur Entwicklungsgeschichte von Centrocestus armatus (Tanabe) mit besonderer Berücksichtigung der Cercariae. Z. Parasitkde, 1938, 10, 293-296.

Yamaguti S. : A synoptical review of life histories of Digenetic Trematodes of Verbetrates. Keigaku Publishing Co Ltd. Yugaku-Sha Ltd, I975, 590 p. + 219 pl. 